\title{
Characterization of wound responsive genes in Aquilaria malaccensis.
}

\begin{abstract}
We report on the isolation and characterization of several genes responsive to wounding in the tropical endangered tree Aquilaria malaccensis. Wounding triggers the formation of a fragrant substance inside the tree stem. Deduced amino acid of the cloned sequences exhibited sequence similarities to their respective homologs: transcription factors of the WRKY gene family (AmWRKY) and $\beta$-1,3-glucanase (AmGLU). A homolog to phenylalanine ammonia-lyase (AmPAL) from previous work was also included. All cDNA sequences were of partial lengths. We studied their expression profiles in a wounding-stress experiment. Mechanical wounding induces AmWRKY in an early response to wounding (3 $\mathrm{h}$ ), and elevates AmPAL and AmGLU expressions after $16 \mathrm{~h}$. It is possible that AmWRKY mediates early wounding response while AmPAL mediates response to fungal infection by co-inducing AmGLU. Their homologs in other plants are known to inhibit fungal growth. Our data provide the first insight into the mechanisms of wounding responses in Aquilaria.
\end{abstract}

Keyword: Agarwood; Aquilaria malaccensis; Genes; Wounding. 